\title{
Pleural Dye Marking Using Radial Endobronchial Ultrasound and Virtual Bronchoscopy before Sublobar Pulmonary Resection for Small Peripheral Nodules
}

\author{
Samy Lachkar ${ }^{a}$ Jean-Marc Baste ${ }^{b}$ Luc Thiberville $^{c}$ Christophe Peillon $^{d}$ \\ Philippe Rinieri $^{d}$ Nicolas Piton ${ }^{\text {e }}$ Florian Guisier $^{c}$ Mathieu Salaun ${ }^{c}$ \\ ${ }^{a}$ Department of Pulmonology and CIC-CRB 1404, Rouen University Hospital, Rouen, France; ${ }^{b}$ Normandie University, \\ UNIROUEN, Inserm U1096, Department of General Thoracic Surgery, Rouen University Hospital, Rouen, France; \\ 'Normandie University, UNIROUEN, QuantIF-LITIS EA 4108, Department of Pulmonology and CIC-CRB 1404, Rouen \\ University Hospital, Rouen, France; d Department of General Thoracic Surgery, Rouen University Hospital, Rouen,

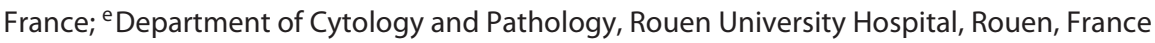

\section{Keywords}

Bronchoscopy · Radial endobronchial ultrasound ·

Peripheral lung nodule · Lung cancer · Minimal invasive thoracic surgery · Dye marking

\footnotetext{
Abstract

Background: Minimally invasive surgery of pulmonary nodules allows suboptimal palpation of the lung compared to open thoracotomy. Objective: The objective of this study was to assess endoscopic pleural dye marking using radial endobronchial ultrasound (r-EBUS) and virtual bronchoscopy to localize small peripheral lung nodules immediately before minimally invasive resection. Methods: The endoscopic procedure was performed without fluoroscopy, under general anesthesia in the operating room immediately before minimally invasive surgery. Then, $1 \mathrm{~mL}$ of methylene blue $(0.5 \%)$ was instilled into the guide sheath, wedged in the subpleural space. Wedge resection or segmentectomy were guided by visualization of the dye on the pleural sur-
}

face. Contribution of dye marking to the surgical procedure was rated by the surgeon. Results: Twenty-five nodules, including 6 ground glass opacities, were resected in 22 patients by video-assisted thoracoscopic wedge resection $(n=$ 11 ) or robotic-assisted thoracoscopic surgery (10 segmentectomies and 1 wedge resection). The median greatest diameter of nodules was $8 \mathrm{~mm}$. No conversion to open thoracotomy was needed. The endoscopic procedure added an average $10 \mathrm{~min}$ to surgical resection. The dye was visible on the pleural surface in 24 cases. Histological diagnosis and free margin resection were obtained in all cases. Median skin-to-skin operating time was $90 \mathrm{~min}$ for robotic segmentectomy and $40 \mathrm{~min}$ for video-assisted wedge resection. The same operative precision was considered impossible by the surgeon without dye marking in 21 cases. Conclusions: Dye marking using r-EBUS and virtual bronchoscopy can be easily and safely performed to localize small pulmonary nodules immediately before minimally invasive resection.

(c) 2018 S. Karger AG, Basel

\section{KARGER}

(c) 2018 S. Karger AG, Basel

E-Mail karger@karger.com

www.karger.com/res
Dr. Samy Lachkar

Department of Pulmonology, Thoracic Oncology, and Respiratory Intensive Care 1 rue de Germont

FR-76031 Rouen Cedex (France)

E-Mail samy.lachkar@chu-rouen.fr 


\section{Introduction}

The diagnosis and management of small pulmonary nodules (SPNs) has become increasingly challenging, with a reported prevalence of SPNs as high as $50 \%$ in lung cancer screening studies [1]. In an era of minimally invasive thoracic surgery (video-assisted thoracoscopic surgery [VATS] or robotic-assisted thoracoscopic surgery [RATS]), subcentimeter lesions or ground glass opacities (GGOs) may be difficult to localize, as these procedures allow only suboptimal palpation of the lung in comparison with open thoracotomy [2-4]. Sometimes, even larger lesions are difficult to find because of their deep location beneath the pleura. Therefore, there is frequent conversion from minimally invasive VATS to more invasive thoracotomy if the nodule cannot be located during the procedure, with a conversion rate as high as $59 \%$ in some studies [5].

To overcome this difficulty, various percutaneous techniques have been developed to localize SPNs, such as computed tomography (CT)-guided radiotracer injection [6], CT-guided wire localization [7], CT-guided fiducial placement and fluoroscopic localization [8], ultrasonography [9], or CT-guided methylene blue injection [10]. Because these percutaneous localization techniques are often performed in radiology departments located far from the operating room (OR), coordination between radiologists, anesthesiologists, and thoracic surgeons is crucial. These logistic issues can be difficult to overcome in certain settings. In addition, the risks of CT-guided transthoracic localization procedures include pneumothorax (16-35\%) and hemorrhage (6-16\%) [11].

Recent studies on pleural dye marking using electromagnetic navigation bronchoscopy (ENB) report accurate nodule localization before minimally invasive resection in a single setting in the OR [12-14].

Another study described a new dye-marking technique using methylene blue and radial endobronchial ultrasound (r-EBUS) [15]. Our objective was to assess a simplified dye-marking technique using a combination of virtual bronchoscopy (VB) and r-EBUS performed immediately before minimally invasive resection of SPNs or GGOs.

\section{Patients and Methods}

This retrospective single-center study was performed at Rouen University Hospital between April 2016 and June 2017. All patients requiring sublobar lung resection (i.e., wedge resection or segmentectomy) for a pulmonary lesion, which was anticipated by the surgeon to be hardly palpable or barely visible intraoperatively, based on its small size, its distance from the pleura, or its GGO appearance on CT, benefited from the endoscopic dye-marking technique. When a solid primary lung cancer was suspected, cases were preferentially submitted to segmentectomy, while cases with GGO or suspicion of metastasis from extrapulmonary cancer were preferentially submitted to wedge resection, according to preoperative multidisciplinary meeting decisions. The study protocol was approved by the Institutional Review Board for Non-Interventional Research of Rouen University Hospital, France (protocol No. E2017-17).

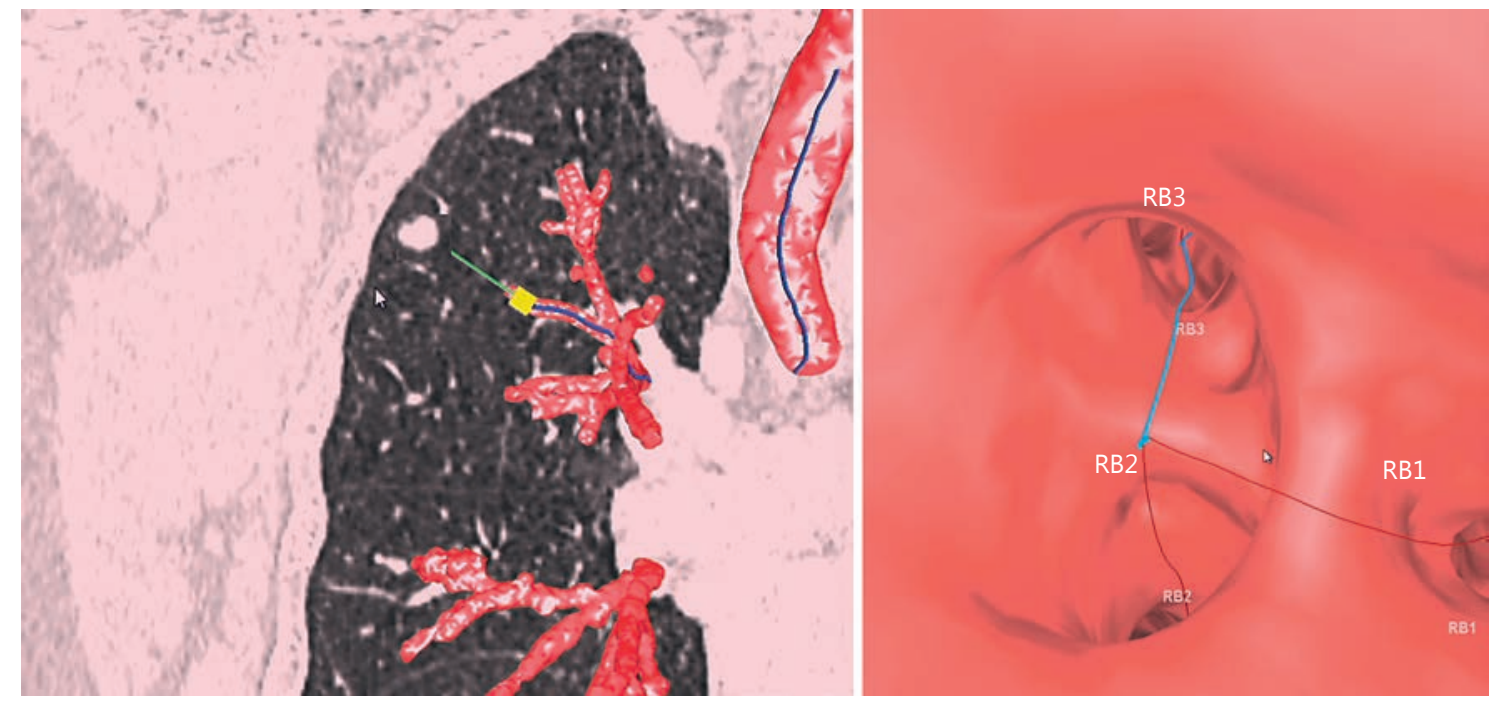

Fig. 1. Virtual bronchoscopy of a 8-mm nodule localized in RB3 (LungPoint ${ }^{\circledR}$ Planner, Broncus Medical Inc., San Jose, CA, USA). Left panel, coronal CT view; right panel, virtual bronchoscopy view. RB, right bronchus. 
Each endoscopic procedure was performed immediately before surgical resection in the same OR. The nodule location was mapped using VB software in order to identify the smallest bronchus leading to the lesion (LungPoint ${ }^{\circledR}$ Planner, Broncus Medical Inc., San Jose, CA, USA) (Fig. 1). Endotracheal ventilation was carried out with a single-lumen endotracheal tube under general anesthesia. The endoscopy was performed using a BF-MP60F endoscope (Olympus ${ }^{\circledR}$, Tokyo, Japan) with a 4 -mm outer diameter and a 2 -mm working channel. The ultrasound probe used was a UMS20-17S radial probe (Olympus ${ }^{\circledR}$ ) with $1.4 \mathrm{~mm}$ diameter that was introduced into the 1.9-mm-diameter guide sheath (Fig. 2). When the fiberscope reached the most distal subsegmental bronchus along the route previously defined in VB, the guide sheath with the $r$-EBUS probe was inserted into the working channel and pushed within the smallest bronchus leading to the lesion in order to reach the subpleural space, without fluoroscopy guidance. The probe was then removed and the guide sheath left in place within the working channel. Subsequently, $1 \mathrm{~mL}$ of methylene blue $(5 \mathrm{mg} /$ $1 \mathrm{~mL}$ ) (Proveblue, Medac, France) was injected into the guide sheath and rinsed with $20 \mathrm{~mL}$ of air in order to clear the methylene blue from the guide sheath.

After the endoscopic procedure, the patient was intubated with a double-lumen tube and appropriately placed in a lateral decubitus position for minimally invasive resection (VATS or RATS) of the lung lesion. On inspection of the lung surface, the methylene blue dye was visualized in order to perform a wedge resection or a segmentectomy using an endoscopic stapler (Covidien Tri-Staple ${ }^{\circledR}$ or Ethicon Echelon Flex ${ }^{\circledR}$ ) (Fig. 3a).

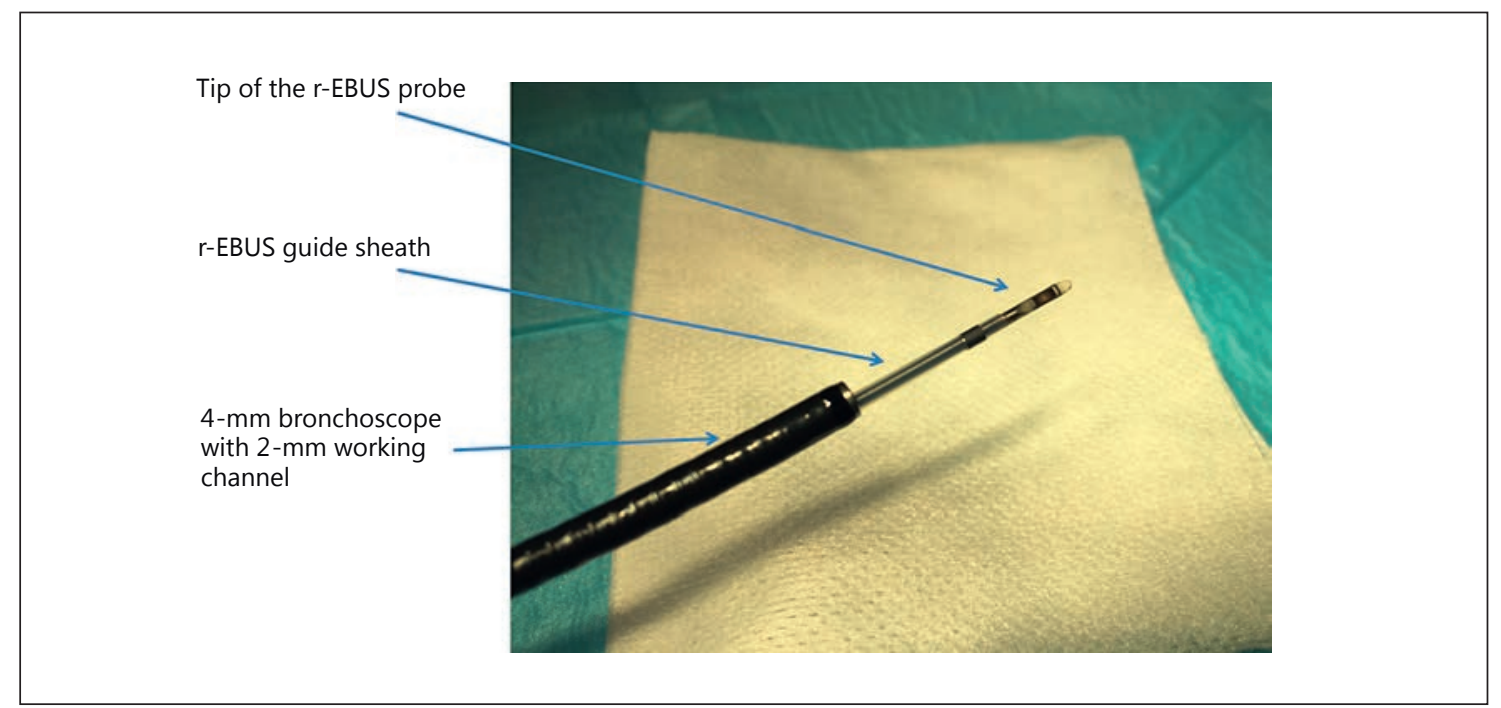

Fig. 2. A radial endobronchial ultrasound probe (r-EBUS; UM-S20-17S, Olympus ${ }^{\circledR}$, Tokyo, Japan) was introduced into the 1.9-mm-diameter guide sheath in the 4-mm-diameter bronchoscope (BF-MP60, Olympus ${ }^{\circledR}$ ).
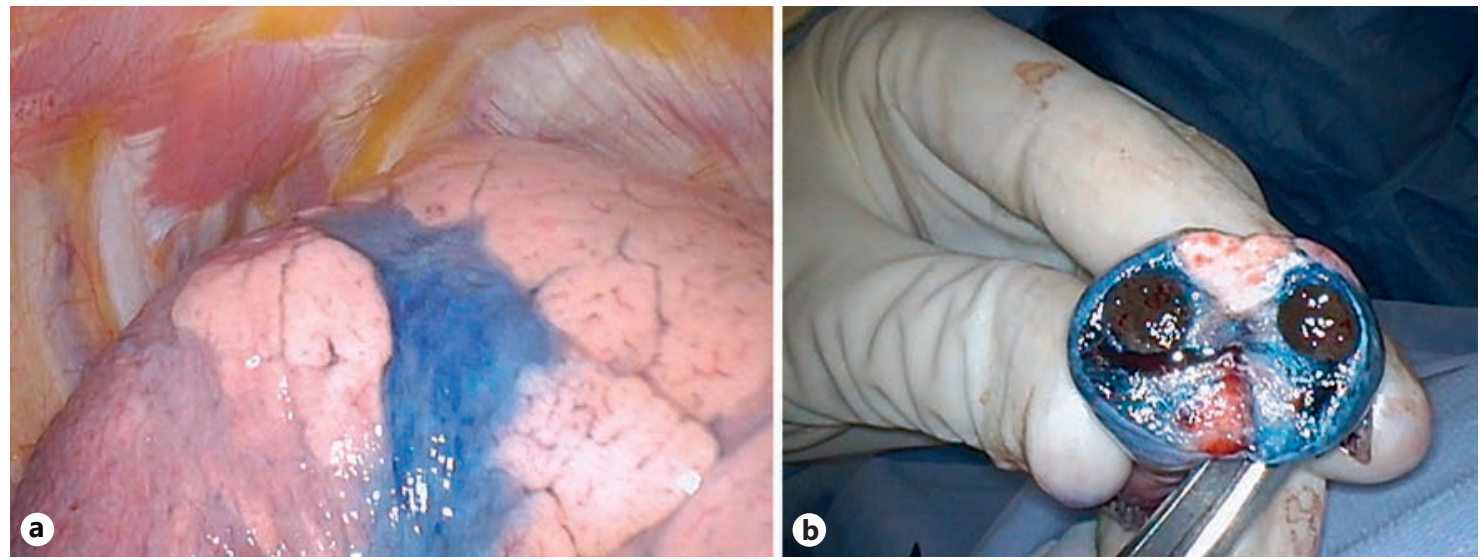

Fig. 3. Perioperative view. a Methylene blue visualized on the pleural surface of the S3 segment during videoassisted thoracoscopic surgery. $\mathbf{b}$ Macroscopic aspect of the nodule right after resection in the operating room: the nodule, an 8-mm melanoma metastasis, was stained with methylene blue. 
Resection of the lesion was guided both by the dye marking and by preoperative high-resolution CT scan for the depth of resection. The nodule resection was immediately evaluated by opening the specimen in the OR prior to pathology analysis (Fig. 3b). The success of the procedure was defined by the presence of the entire lesion with tumor-free margins in the resection specimen on histological analysis.

The entire resection procedure was recorded (video). The contribution of dye marking to the procedure was assessed retrospectively by the surgeon on the basis of the recorded video sequences, according to the grading system published by Sato et al. [16]: grade A, the same level of operative precision was considered to be impossible without marking; grade B, a similar level of precision was considered to be possible, but marking enabled confident performance of the operation, and grade $\mathrm{C}$, the same operation was considered to be possible without marking.

Collected data included demographic information, the size of the lesion and distance from the pleural surface, radiographic characteristics of the lesion (solid vs. ground glass), contribution of the dye-marking technique, perioperative data, adverse events, type or resection needed for diagnosis, and final pathologic diagnosis.

\section{Results}

\section{Patients' Characteristics}

Twenty-two consecutive patients (11 men, 11 women; mean age 61.5 [47-70] years) with 25 lesions were included. Sixteen patients (73\%) had a substantial smoking history, and 10 patients had a history of malignancy ( 2 with lung cancer and 8 with extrathoracic cancer).

\section{Characteristics of the Lung Lesions}

The diagnosis of the nodule was unknown preoperatively in all patients. Nineteen out of 25 nodules (72\%) were solid and 6 were GGOs (Table 1). The median greatest diameter of the nodule was $8(3-15) \mathrm{mm}$, and in $23 / 25$ (92\%) of the cases, the nodule diameter was $<10 \mathrm{~mm}$. The median distance between the center of the lesion and the nearest parietal pleural surface was $10(2-27) \mathrm{mm}$ (Table 1). The lesions were located in the upper lobe in 17 out of 25 cases $(68 \%)$ (Table 1$)$.

\section{Perioperative and Postoperative Outcomes}

These results are displayed in Table 2. No complications due to dye marking were reported. The mean time of the endoscopic procedure, including injection of the methylene blue dye, was $10(5-15) \mathrm{min}$. There was no difference in terms of size, distance from the pleura, solid nature or GGO, or bronchus signs between the 12 nodules that were visualized using r-EBUS and the 13 that were not (Table 3 ). A bronchus sign was seen in only 2
Table 1. Characteristics of the pulmonary nodules $(n=25)$

\begin{tabular}{ll}
\hline Type of nodule, solid/GGO & $19 / 6$ \\
Median greatest diameter (min.-max.), mm & $8(3-15)$ \\
Median distance between nodule and pleura & \\
(min.-max.), mm & $10(2-27)$ \\
\hline Location of the nodule & \\
Right upper lobe & $14(56 \%)$ \\
RB1 & 9 \\
RB2 & 1 \\
RB3 & 4 \\
Left upper lobe & $3(12 \%)$ \\
LB1 & 2 \\
LB5 & 1 \\
Right lower lobe & $6(24 \%)$ \\
RB6 & 1 \\
RB8 & 2 \\
RB9 & 2 \\
RB10 & 1 \\
Left lower lobe & $2(8 \%)$ \\
LB7 & 1 \\
LB10 & 1 \\
\hline
\end{tabular}

min.-max., minimum-maximum; RB, right bronchus; LB, left bronchus; GGO, ground glass opacities.

cases, and an ultrasound image of the nodule was found in only 12 cases $(48 \%)$.

The mean time between endoscopic dye marking and visualization of the lesion by the surgeon was $37(20-60)$ min. This included the change of tracheal tube, the positioning of the patient, the preparation of equipment for VATS or RATS, and the thoracoscopic examination of the pleural surface.

Fifteen lesions were resected by wedge using VATS in 14 cases and RATS in 1 case. All 10 segmentectomies were performed using RATS.

In all but 1 of the 25 cases (96\%), the dye was visible on the pleural surface. The contribution of dye marking was rated by the surgeon as grade A in 21 cases and as grade $B$ in 3 cases.

For robotic segmentectomy, the median operating time (skin to skin) and the OR time were $90(80-170)$ and 210 (210-260) $\mathrm{min}$, respectively. The median operating time (skin to skin) and the OR time of video-assisted wedge resections were 40 (25-70) and 120 (90-200) min, respectively. No conversion to open thoracotomy was needed.

A definitive diagnosis was obtained by pathological evaluation in all the 25 nodules. This included 11 with primary lung cancer, 6 with metastatic cancer, and 8 benign nodules (4 granulomas, 3 inflammations, and $1 \mathrm{am}$ - 
Table 2. Perioperative and postoperative outcomes

Table 3. Nodule features according to ultrasound results
Mean navigation time (min.-max.), min

Mean time between dye marking and surgery

$$
\text { (min.-max.), min }
$$

Median operating time, skin to skin (min.-max.), min Video-assisted wedge resection

Robotic segmentectomy

Median operating room time (min.-max.), min

Video-assisted wedge resection

Robotic segmentectomy

Type of surgery

VATS

RATS

Type of resection

Wedge resection

Segmentectomy

Surgeon rating of dye marking

Grade A

Grade B

Grade C

Definitive diagnosis

Malignant

Lung cancers

Gastric cancer metastases

Melanomas

Lymphomas

Kidney cancer

Benign

Granulomas

Inflammations

Amyloidosis

Surgical complication

Conversion to open surgery $\quad 0$

Other complications

Median length of hospital stay (min.-max.), days

min.-max., minimum-maximum; VATS, video-assisted thoracoscopic surgery; RATS, robotic-assisted thoracoscopic surgery.

\begin{tabular}{llcl}
\hline & \multicolumn{2}{c}{ Ultrasound image } & \multirow{2}{*}{$p^{*}$} \\
\cline { 2 - 3 } & Yes: 12(48\%) & No: 13 (52\%) & \\
\hline Solid nodule & 10 & 9 & 0.64 \\
GGO & 2 & 4 & \\
$\begin{array}{l}\text { Median size (min.-max.), mm } \\
\text { Median distance between nodule and pleura }\end{array}$ & $8(5-15)$ & $8(3-10)$ & 1 \\
$\quad$ (min.-max.), mm & $10(2-35)$ & $10(2-27)$ & 1 \\
Bronchus signs & 2 & 0 & 0.22 \\
\hline
\end{tabular}

GGO, ground glass opacities; min.-max., minimum-maximum. * Fisher exact test.
$10(5-15)$

$37(30-45)$

$40(25-70)$

$90(80-170)$

$120(90-200)$

$210(210-260)$

$11(50 \%)$

$11(50 \%)$

15

10

21

3

1

17

11

2

3

0

$3(1-8)$

Immediately before Sublobar Surgery 
yloidosis). All malignant lesions were completely resected with negative microscopic resection margins.

The median length of hospital stay was 3 days, with a prolonged air leak in 2 cases leading to an extended stay of 7 and 8 days, respectively. No other complications occurred during the hospitalization.

\section{Discussion}

In this series, we showed that VB and r-EBUS for methylene blue marking of SPNs appear easy and safe to use and efficiently target the area of sublobar resection. The mean navigation time was low (10 $\mathrm{min})$, and a single operative setting was used for both the dye-marking technique and for the surgical procedure. There were no complications related to the preoperative procedure in the present series. All nodules were successfully localized and fully resected with tumor-free margins in all malignant lesions.

Importantly, our dye-marking technique was considered useful by the surgeon in the majority of cases. Moreover, we recorded a median operating time (skin to skin) for RATS segmentectomy of $90 \mathrm{~min}$, which appears lower than in our previous series without marking (140 min) [17]. Similarly, we report a median operating time for VATS wedge resection of $40 \mathrm{~min}$, compared to $69 \mathrm{~min}$ in other studies $[18,19]$. Furthermore, the overall occupation time of the OR in our series for VATS wedge resection (125 $\mathrm{min}$ ) also compared favorably with previous reports (150 min) [20].

To date, CT-guided wire placement (including hook wires, microcoils, and spiral wires) is the most common procedure used to localize small, nonpalpable pulmonary lesions, with a high rate of successful localization [11]. However, this technique has several limitations, including pneumothorax (16-35\%) and hemothorax (6-16\%) $[7,11,21,22]$, as well as dislodgement of the guide wire, which can lead to the failure of localization. A few cases of air embolism have also been reported [22-24]. CTguided localization can be difficult to implement with a reported median procedure time of about $40 \mathrm{~min}$ in awake patients [25]. CT-guided wire placement is performed in a dual setting, as the patient must be transferred from the radiology suite to the OR for the surgical procedure. Preoperative transthoracic CT-assisted methylene blue staining has recently shown a good sensitivity for small nodule localization. The procedure is less costly than wire-related techniques and obviates wire-related complications, but the coloration of the targeted area ap- pears significantly affected by the time between tumor marking and thoracoscopy $[26,27]$.

Few data have been published on bronchoscopy dyemarking techniques, most of them concerning ENB-guided localization followed by minimally invasive resection [12-14]. r-EBUS associated with a VB dye-marking technique uses a thinner bronchoscope $(4 \mathrm{~mm})$ compared to ENB $(6 \mathrm{~mm})$, which results in a more distal progression of the endoscope and a more selective catheterization of the subsegmental bronchus. r-EBUS also appears to be more cost-effective than ENB, which requires specific preparation of the OR and more expensive disposable material. On average, r-EBUS costs 5 times less than ENB.

The technique presented here alleviates the loss of accuracy linked to the resorption of methylene, as both the mean time of the dye-marking technique and the time to surgical localization of the lesion are very low. Another advantage of our dye-marking technique is that there is no exposure to radiation as fluoroscopy was not used [28], whereas fluoroscopy was used in the 3 published studies using ENB [12-14].

As opposed to a recent study [15], a characteristic EBUS image of the peripheral nodule was obtained in only 12 cases (48\%), whereas methylene blue was visible at the surface of the pleura in $95 \%$ of the cases in our series. This may be explained by the large proportion of GGOs among the patients explored (28\%), by the very small size of the lesions, and by the absence of a bronchus sign in $92 \%$ of the cases. This suggests that VB may be the most useful part of the technique, while the role of r-EBUS may be essentially that of a guide to reach and to visualize the pleural surface. It is possible that future studies will demonstrate that a simplified procedure using only $\mathrm{VB}$ and a catheter, without r-EBUS or other imaging devices, is as accurate as the procedure presented in this study.

Sato et al. $[16,29]$ also reported the use of VB and a bronchoscopic multi-spot dye-marking technique using 3-dimensional virtual imaging for precise thoracoscopic sublobar lung resection with safe surgical margins. However, their procedure appears difficult to implement, as the procedure was not performed immediately before the surgery or during the same surgical procedure but 2 days preoperatively. Therefore, a second CT scan had to be performed to confirm the location of markings within 2 hours of the mapping procedure [16]. The authors also reported a mean time of $59 \mathrm{~min}$ for mapping and $20 \mathrm{~min}$ for the bronchoscopy.

In our series, a malignant lesion was diagnosed in $61 \%$ of patients, which appears higher than in a reported series of sublobar resection of pulmonary nodules, with a prev-
$6 \quad$ Respiration DOI: $10.1159 / 000486205$
Lachkar/Baste/Thiberville/Peillon/Rinieri/ Piton/Guisier/Salaun 
alence of malignancy varying from 6 to $28 \%$ for solid nodules of $5-10 \mathrm{~mm}$ in size and from 59 to $73 \%$ for GGOs [30]. The high rate of malignancy reported in our series may be explained by the size of the present series. It may also reflect the selection of patients based on multidisciplinary meeting decisions, including patients with either a nodule detected during CT screening or a metastatic nodule detected during follow-up for other cancers.

Besides its retrospective nature, the principal limitation of our study is related to a possible selection bias as procedures were performed by a single surgical team. Moreover, the absence of a control group did not allow any comparison of either the operating times or the rates of thoracotomy among patients with similar nodules that were not dye marked before minimally invasive surgery. However, we believe that the results presented here compare favorably with previous techniques designed to help surgical resection during video- or robotic-assisted sublobar resections.

\section{Conclusion}

Methylene blue dye marking using r-EBUS and VB seems to be an easy and safe technique to guide minimally invasive resection of small peripheral lung nodules and GGOs.

\section{Acknowledgement}

We are grateful to Nikki Sabourin-Gibbs, Rouen University Hospital, for editing the manuscript.

\section{Financial Disclosure and Conflicts of Interest}

The authors declare no conflict of interest related to the submitted work. The authors have reported that no funding was received for this study.

\section{References}

1 Ost D, Fein AM, Feinsilver SH: Clinical practice. The solitary pulmonary nodule. $\mathrm{N}$ Engl J Med 2003;348:2535-2542.

2 Gould MK, Donington J, Lynch WR, Mazzone PJ, Midthun DE, Naidich DP, Wiener RS: Evaluation of individuals with pulmonary nodules: when is it lung cancer? Diagnosis and management of lung cancer, 3rd ed: American College of Chest Physicians evidence-based clinical practice guidelines. Chest 2013;143:e93S-e120S.

-3 Detterbeck FC: Lobectomy versus limited resection in T1N0 lung cancer. Ann Thorac Surg 2013;96:742-744.

4 Zaman M, Bilal H, Woo CY, Tang A: In patients undergoing video-assisted thoracoscopic surgery excision, what is the best way to locate a subcentimetre solitary pulmonary nodule in order to achieve successful excision? Interact Cardiovasc Thorac Surg 2012; 15:266-272.

5 Cardillo G, Regal M, Sera F, Di Martino M, Carbone L, Facciolo F, Martelli M: Videothoracoscopic management of the solitary pulmonary nodule: a single-institution study on 429 cases. Ann Thorac Surg 2003;75:16071611; discussion 1611-1602.

6 Grogan EL, Jones DR, Kozower BD, Simmons WD, Daniel TM: Identification of small lung nodules: technique of radiotracer-guided thoracoscopic biopsy. Ann Thorac Surg 2008; 85:S772-S777.

7 Chen S, Zhou J, Zhang J, Hu H, Luo X, Zhang Y, Chen H: Video-assisted thoracoscopic solitary pulmonary nodule resection after CT- guided hookwire localization: 43 cases report and literature review. Surg Endosc 2011;25: 1723-1729.

8 Sancheti MS, Lee R, Ahmed SU, Pickens A, Fernandez FG, Small WC, Nour SG, Force SD: Percutaneous fiducial localization for thoracoscopic wedge resection of small pulmonary nodules. Ann Thorac Surg 2014;97: 1914-1918; discussion 1919.

-9 Wicky S, Mayor B, Cuttat JF, Schnyder P: CTguided localizations of pulmonary nodules with methylene blue injections for thoracoscopic resections. Chest 1994;106:1326-1328.

10 Matsumoto S, Hirata T, Ogawa E, Fukuse T, Ueda H, Koyama T, Nakamura T, Wada H: Ultrasonographic evaluation of small nodules in the peripheral lung during video-assisted thoracic surgery (VATS). Eur J Cardiothorac Surg 2004;26:469-473.

-11 Park CH, Han K, Hur J, Lee SM, Lee JW, Hwang SH, Seo JS, Lee KH, Kwon W, Kim $\mathrm{TH}$, Choi BW: Comparative effectiveness and safety of preoperative lung localization for pulmonary nodules: a systematic review and meta-analysis. Chest 2017;151:316-328.

$\checkmark 12$ Awais O, Reidy MR, Mehta K, Bianco V, Gooding WE, Schuchert MJ, Luketich JD, Pennathur A: Electromagnetic navigation bronchoscopy-guided dye marking for thoracoscopic resection of pulmonary nodules. Ann Thorac Surg 2016;102:223-229.

13 Luo K, Lin Y, Lin X, Yu X, Wen J, Xi K, Lin P, Zhang L: Localization of peripheral pulmonary lesions to aid surgical resection: a novel approach for electromagnetic navigation bronchoscopic dye marking. Eur J Cardiothorac Surg 2017;52:516-521.

14 Bolton WD, Howe H 3rd, Stephenson JE: The utility of electromagnetic navigational bronchoscopy as a localization tool for robotic resection of small pulmonary nodules. Ann Thorac Surg 2014;98:471-475; discussion 475-476.

15 Liu Z, Yang R, Shao F, Pan Y: Localization of small peripheral pulmonary lesion by methylene blue injection with radial endobronchial ultrasonography in sublobar resection. Ann Thorac Surg 2016;101:e57-e59.

16 Sato M, Yamada T, Menju T, Aoyama A, Sato T, Chen F, Sonobe M, Omasa M, Date H: Virtual-assisted lung mapping: outcome of 100 consecutive cases in a single institute. Eur J Cardiothorac Surg 2015;47:e131-e139.

17 Rinieri P, Peillon C, Salaun M, Mahieu J, Bubenheim M, Baste JM: Perioperative outcomes of video- and robot-assisted segmentectomies. Asian Cardiovasc Thorac Ann 2016;24:145-151.

18 Lin L, Hu D, Zhong C, Zhao H: Safety and efficacy of thoracoscopic wedge resection for elderly high-risk patients with stage I peripheral non-small-cell lung cancer. J Cardiothorac Surg 2013;8:231.

19 David G, Gunnarsson CL, Moore M, Howington J, Miller DL, Maddaus MA, McKenna RJ Jr, Meyers BF, Swanson SJ: Surgeons' volume-outcome relationship for lobectomies and wedge resections for cancer using videoassisted thoracoscopic techniques. Minim Invasive Surg 2012;2012:760292. 
20 Swanson SJ, Miller DL, McKenna RJ Jr, Howington J, Marshall MB, Yoo AC, Moore M, Gunnarsson CL, Meyers BF: Comparing robot-assisted thoracic surgical lobectomy with conventional video-assisted thoracic surgical lobectomy and wedge resection: results from a multihospital database (Premier). J Thorac Cardiovasc Surg 2014;147:929-937.

- 21 Dendo S, Kanazawa S, Ando A, Hyodo T, Kouno Y, Yasui K, Mimura H, Akaki S, Kuroda M, Shimizu N, Hiraki Y: Preoperative localization of small pulmonary lesions with a short hook wire and suture system: experience with 168 procedures. Radiology 2002; 225:511-518.

$\checkmark 22$ Horan TA, Pinheiro PM, Araujo LM, Santiago FF, Rodrigues MR: Massive gas embolism during pulmonary nodule hook wire localization. Ann Thorac Surg 2002;73:1647-1649.

-23 Ichinose J, Kohno T, Fujimori S, Harano T, Suzuki S: Efficacy and complications of computed tomography-guided hook wire localization. Ann Thorac Surg 2013;96:12031208.
24 Suzuki K, Shimohira M, Hashizume T, Ozawa Y, Sobue R, Mimura M, Mori Y, Ijima $\mathrm{H}$, Watanabe K, Yano M, Yoshioka H, Shibamoto Y: Usefulness of CT-guided hookwire marking before video-assisted thoracoscopic surgery for small pulmonary lesions. J Med Imaging Radiat Oncol 2014;58:657-662.

25 Gonfiotti A, Davini F, Vaggelli L, De Francisci A, Caldarella A, Gigli PM, Janni A: Thoracoscopic localization techniques for patients with solitary pulmonary nodule: hookwire versus radio-guided surgery. Eur J Cardiothorac Surg 2007;32:843-847.

26 McConnell PI, Feola GP, Meyers RL: Methylene blue-stained autologous blood for needle localization and thoracoscopic resection of deep pulmonary nodules. J Pediatr Surg 2002; 37:1729-1731.
27 Lenglinger FX, Schwarz CD, Artmann W: Localization of pulmonary nodules before thoracoscopic surgery: value of percutaneous staining with methylene blue. AJR Am J Roentgenol 1994;163:297-300.

28 Jain P, Fleming P, Mehta AC: Radiation safety for health care workers in the bronchoscopy suite. Clin Chest Med 1999;20:33-38, ix-x.

29 Sato M, Omasa M, Chen F, Sato T, Sonobe M, Bando T, Date H: Use of virtual assisted lung mapping (VAL-MAP), a bronchoscopic multispot dye-marking technique using virtual images, for precise navigation of thoracoscopic sublobar lung resection. J Thorac Cardiovasc Surg 2014;147:1813-1819.

30 Wahidi MM, Govert JA, Goudar RK, Gould MK, McCrory DC; American College of Chest Physicians: Evidence for the treatment of patients with pulmonary nodules: when is it lung cancer?: ACCP evidence-based clinical practice guidelines (2nd edition). Chest 2007; 132:94S-107S. 